\title{
EL EFECTO DE SEQUÍA EN EL CRECIMIENTO, LA FOTOSÍNTESIS Y LA INTERCEPCIÓN DE LUZ EN FRIJOL COMÚN1
}

\author{
Abelardo Nuñez Barrios ${ }^{2}$, Joe Ritchie ${ }^{3}$, Alvin J.M. Smucker ${ }^{3}$
}

\begin{abstract}
RESUMEN
El efecto de sequía en el crecimiento, la fotosíntesis y la intercepción de luz en frijol cumún. Se investigó el impacto del estrés hídrico en el crecimiento foliar y en la intercepción de luz, la conductancia estomática y producción de materia seca en frijol. En la Universidad Estatal de Michigan se establecieron tratamientos de sequía en diferentes etapas fenológicas: etapa vegetativa (EV), inicio de floración (IF) y llenado de las semillas (LS). El déficit hídrico disminuyó rápidamente el índice de área foliar (IAF), siendo mayor la reducción en el tratamiento IF lo que causó una disminución significativa en la intercepción de la luz. La conductancia estomática y la fotosíntesis se redujeron también bajo el estrés hídrico pero las tasas de fotosíntesis sólo disminuyeron significativamente cuando la apertura estomática estuvo por debajo de $0,8 \mathrm{~cm}$ /seg. La producción de materia seca estuvo altamente relacionada con la intercepción de la luz aunque, a la misma cantidad de luz interceptada, la producción de materia seca en IF fue menor en un $6,5 \%$ en comparación con el testigo.
\end{abstract}

\begin{abstract}
Effect of drought on photosynthesis, growth, and light interception in dry beans. The effect of water deficit on leaf area index, light interception, stomatal conductance, and dry matter production in dry beans was studied in this investigation carried out at Michigan State University, where drought treatments were imposed at different phenological stages such as vegetative (VS), initial flowering (IF) and seed fiJling (SF). Water stress rapidly reduced the Jeaf area index, mostly in IF, causing a significant decrease in light interception. Stomatal conductance and photosynthesis were also reduced by water deficit. However, the $\mathrm{CO}_{2}$ assimilation rate had a significant decrease only when stomatal conductance was lower than $0.8 \mathrm{~cm} / \mathrm{sec}$. Dry matter production was cJosely related to light interception. Nevertheless, at the same amount of intercepted radiation, production of dry matter in IF lagged behind the control by $6.5 \%$.
\end{abstract}

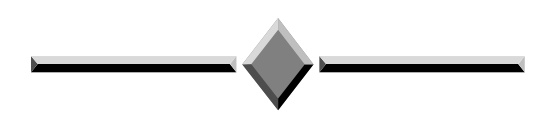

\section{INTRODUCCIÓN}

Las sequías recurrentes afectan la producción de frijol no solo en regiones semiáridas y en el trópico seco, sino también en regiones de clima templado con veranos relativamente cálidos. Sin embargo la magnitud del efecto de la sequía depende de la intensidad y la duración de ésta, así como de la etapa fenológica en que se encuentre el cultivo. El déficit hídrico en etapas tempranas generalmente afectan el alargamiento y el tamaño final de las hojas, en cambio en estadios más avanzados se incrementa la senescencia foliar y la pérdida de follaje (Nuñez y Foster, 1996; Kramer, 1983). Esta disminución del área foliar, conjuntamente con una reducción en la conductancia estomática, pueden limitar tanto la intercepción de la energía solar como la tasa de fotosíntesis y finalmente la producción de materia seca en la planta (Dai et al., 1992; Hall y Shultz, 1980). Por otro lado, existe un cuestionamiento creciente en cuanto al efecto real de los movimientos estomáticos inducidos por el déficit hídrico, en relación con la fotosíntesis y la producción de materia seca en un cultivo. Farqhuar y Sharkey (1982), concluyeron, después de una extensa revisión bibliográfica, que aparte de algunos efectos de tipo hormonal como el producido por el ácido abscísico, el cierre estomático es rara vez la principal causa de la reducción en materia seca que se observa al disminuir la disponibilidad de agua en el suelo. Estudios posteriores han demostrado, que aún bajo sequías relativamente severas, la capacidad fotosintética del mesófilo de la lámina foliar no es fácilmente reducida (Castonguay y Markhart, 1991) y las tasas de fotosíntesis sólo disminuyen radicalmente cuando por sequía se pierde más

\footnotetext{
1 Parte de la tesis de doctorado llevada a cabo en la Univeridad Estatal de Michigan.

2 Centro de Investigación Regional Norte-Centro Ave. Cuauhtemoc 2000, 4to piso Col. Cuauhtemoc 3 I 020, Chihuahua, Chih. México.

3 Michigan State University East Lansing, Michigan.
} 
del $40 \%$ del contenido de clorofila en la hoja (Congreso Nacional de Biotecnología, 1996).

El objetivo del presente trabajo fue investigar el efecto de la sequía edáfica en diferentes estados fenológicos en el área foliar, la conductancia estomática, la fotosíntesis, la intercepción de luz y la producción de materia seca en el cultivo del frijol.

\section{MATERIALES Y MÉTODOS}

El estudio se llevó a cabo en condiciones de campo en la estación experimental de la Universidad Estatal de Michigan (Kellogg Biological Station) durante el verano de 1988 y 1989. El clima durante el verano en esta localidad se caracteriza por tener humedades relativas promedio de entre 75 y $80 \%$ y temperaturas máximas entre 34 y $36^{\circ} \mathrm{C}$. El experimento se estableció bajo una estructura metálica computarizada (rainout shellter) que utiliza un sensor de humedad y se cierra automáticamente al inicio de cualquier evento de lluvia y que abre también automáticamente mediante un sensor de radiación solar. De tal manera se puede ejercer un control total de los períodos de sequía que se necesiten establecer, sin modificar significativamente el microclima dentro de la estructura. Para preparar el experimento, se quemó la hierba y se rastreó para posteriormente sembrar la variedad de frijol (Phaseolus vulgaris L. cv Seafarer) de tipo erecto, grano blanco y ciclo corto (85 días a la madurez) en parcelas de cuatro surcos de seis metros de largo. El suelo es arenoso (Psammentic Hapludalt) de una profundidad promedio de $1,5 \mathrm{~m}$ y con contenidos de materia orgánica por debajo de $1 \%$. La siembra se llevó a cabo manualmente el 5 y el 10 de junio en 1988 y 1989, respectivamente, utilizando un exceso de semillas para ralear posteriormente a una densidad de 16 plantas $/ \mathrm{m}^{2}$. La fertilización se realizó también manualmente, aplicándose una dosis de fertilizante de 120, 100 y $100 \mathrm{~kg} / \mathrm{ha}$ de nitrógeno, fósforo y potasio respectivamente, dosis recomendadas por el servicio de extensión agrícola de la Universidad para esa zona. Se utilizó un sistema de irrigación por aspersión, descrito ya por Nesmith et al. (1990), que fue puesto en operación cada dos o tres días para suministrar una lámina de riego aproximada de 20 a 24 mmlsemana, suficiente para reponer las pérdidas de agua por evapotranspiración y conservar el suelo a capacidad de campo, valor que en este tipo de suelo representa un contenido volumétrico de agua de entre 0,15 y $0,17 \mathrm{~cm}^{3} / \mathrm{cm}^{3}$. En 1988 los tratamientos de sequía se establecieron a los 15 y 29 DDS lo que corresponde a una sequía que comenzó en la etapa vegetativa de rápido crecimiento $(\mathrm{EV})$ e inicio de floración (IF) respectivamente. En el segundo año se repitió el tratamiento de sequía al inicio de flora- ción y se anexó uno más a los 45 DDS, para cubrir la etapa de llenado de la semilla (LS). Todos los tratamientos de sequía fueron terminales, esto es que al suspenderse el riego a los 15-29 y 29-45 DDS no se volvió a suministrar agua durante el resto del ciclo vegetativo lo que propició sequías de diferente intensidad y duración. Como testigo se utilizó un tratamiento de riego al que se le suministró agua durante todo el experimento para mantenerlo a capacidad de campo. El contenido de humedad del suelo se midió con un dispersor de neutrones bajando la fuente de energía a través de tubos de aluminio colocados en el centro de cada unidad experimental. Las lecturas se realizaron cada dos semanas a seis diferentes profundidades $(0,25,0,50,0,75,1,00,1,25$ y 1,50 m) en todos los tratamientos.

Las mediciones de conductancia estomática y fotosíntesis sólo se tomaron en el testigo y el tratamiento de sequía IF, utilizándose un analizador ADC infrarrojo de gases (Analytical Development Company, Haddesdon, U.K.). Para estas mediciones se seleccionaron al azar, tres hojas completamente expuestas a la radiación solar tomándose la lectura con las hojas orientadas perpendicularmente a los rayos solares para obtener posteriormente, una media por tratamiento. El instrumento se calibró antes de cada día de mediciones basándose en una concentración conocida de bióxido de carbono (300 ppm). Las medidas se tomaron entre las 10 y 12 hrs EDT a los 36,46, y 55 DDS en 1988 y a los 33,42 y 52 DDS durante 1989. La intercepción de luz se midió en 1989 alrededor del medio día utilizando el método de Adams y Arkin (1977). Las observaciones con este método se hicieron semanalmente comenzando a los 25 DDS, colocando la regla métrica transversalmente sobre los surcos en la parte central de cada unidad experimental en la cual se tomaron cinco muestras por parcela lo que corresponde aproximadamente a una superficie de $1 \mathrm{~m}^{2}$, La radiación fotosintéticamente activa fue estimada tomando el $48 \%$ de la radiación solar (Monteith, 1977) registrada en la estación climatológica localizada a $50 \mathrm{~m}$ del sitio experimental. El área foliar (AF) se tomó basándose en muestreos semanales de segmentos de surcos de $0,25 \mathrm{~m}$ de los cuales se hizo un muestreo de las plantas correspondientes midiendo por cada hoja largo (L) y ancho (A) para posteriormente usar la fórmula $\mathrm{AF}=0,603+0,581$ (LxA). Esta ecuación fue estimada previamente para esta variedad usando la longitud y el ancho de diferentes hojas versus las lecturas de un medidor electrónico de área foliar.

La senescencia foliar se cuantificó basándose en el porcentaje en peso seco de hojas completamente amarillas o necróticas en comparación con el resto de hojas verdes fotosintéticamente activas. Al final del ciclo, se cosecharon los dos surcos centrales colectándose tanto hojas como tallos y órganos reproductivos los cuales 
fueron colocados en bolsas de papel y puestos en una estufa de secado a $70^{\circ} \mathrm{C}$ por 72 horas para después ser pesados en una balanza de precisión. El análisis estadístico para todas las lecturas se llevó a cabo utilizando un diseño de bloques al azar con tres repeticiones.

\section{RESULTADOS Y DISCUSIÓN}

En el primer año de este estudio los tratamientos de sequía comenzaron durante la etapa vegetativa y el inicio de floración. En EV, el contenido de agua disminuyó paulatinamente en el perfil del suelo siendo mayor la pérdida en los primeros $0,50 \mathrm{~m}$ (Figura. 1B) donde se llegó a valores cercanos al punto de marchitamiento permanente, esto es, valores estimados de 0,06 y 0,08 $\mathrm{cm}^{3} / \mathrm{cm}^{3}$ para profundidades de 0,25 y $0,25 \mathrm{~m}$ respectivamente. Para el tratamiento IF los patrones de extracción de agua fueron un poco diferentes. La reducción del contenido de agua en el suelo en los primeros 30 días después del inicio de la sequía fue mayor que los observados en EV en el mismo lapso de tiempo (Figura 1 C), además, entre los 57 y 71 DDS la mayor parte de la absorción de agua en el tratamiento IF ocurrió por debajo de un metro de profundidad.

En el segundo año, el tratamiento de IF tuvo un patrón de extracción de agua semejante a ese del primero. Esto es, hubo una rápida absorción en los primeros 0,70 $\mathrm{m}$ seguido por una mayor absorción de agua de capas más profundas (Figura 2C). En el tratamiento LS, el contenido de humedad en el suelo en el primer metro de profundidad disminuyó aún más rápido que en el tratamiento IF y de igual manera, al final del ciclo, la mayor cantidad de agua aprovechable por la planta se encontró a profundidades mayores de $0,75 \mathrm{~cm}$. En el testigo, el contenido de humedad se conservó cerca de capacidad de campo en todo el perfil, como se muestra en la Figura 2A.

El efecto de la sequía sobre el índice de área foliar (IAF) se observó poco días después de haberse iniciado ésta (Figura. 3A y B). En los tratamientos IF en ambos años, se puede ver una rápida reducción en el IAF después de dos semanas de iniciada la sequía alcanzando este tratamiento a los 50 DDS, un $37 \%$ menos de área foliar en comparación con el testigo. En el tratamiento LS se observó un efecto similar donde a los 71 DDS el índice del área foliar se redujo en casi un 50\% en comparación con el tratamiento irrigado (Figura 3B). Por otro lado, la respuesta del EV al déficit hídrico resultó en un aparente preacondicionamiento de la planta a la sequía la cual afectó más el tamaño de las hojas que la pérdida de éstas por senescencia. El tamaño de las hojas que iniciaron su crecimiento después del estrés fue en promedio de $60 \mathrm{~cm}^{2} /$ hoja en el EV en comparación con $138 \mathrm{~cm}^{2} /$ hoja del testigo. Morgan, (1984) informa que al someter a sequía plantas jóvenes de trigo, éstas incrementaron significativamente la acumulación de solutos en sus células, y aunque alcanzaron menor tamaño se incrementó su tolerancia a la deshidratación de sus tejidos. Por otro lado se ha observado que, aún a expensas del rendimiento, las rápidas tasas de senescencia son también un mecanismo que le permite a la planta sobrevivir a periodos largos de sequía (Laing, et al. 1983).

La conductancia estomática conjuntamente con la fotosíntesis son mediciones que se llevaron a cabo en el

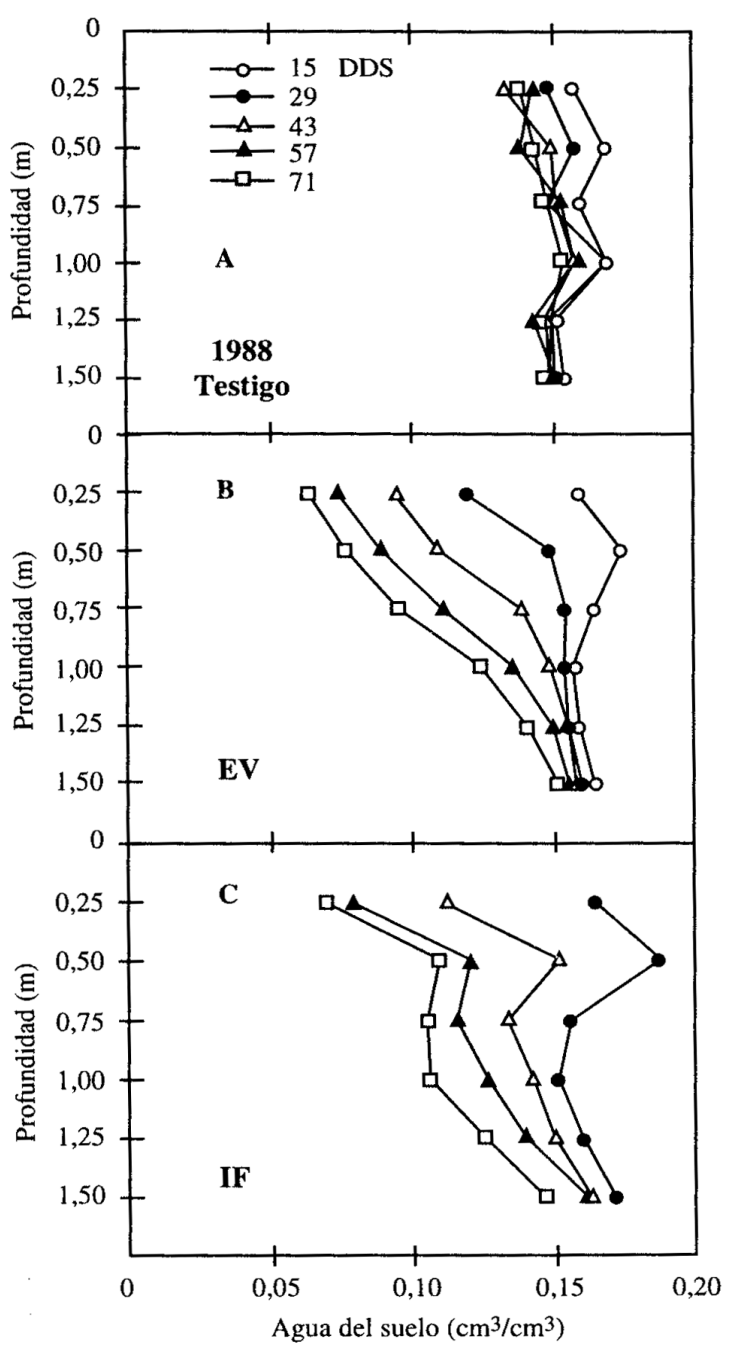

Figura. 1. Cambios en el contenido volumétrico de agua en los tratamientos de sequía iniciados en la etapa vegetativa (EV) y floración (IF) en contraste con el testigo. KBS, Michigan. 


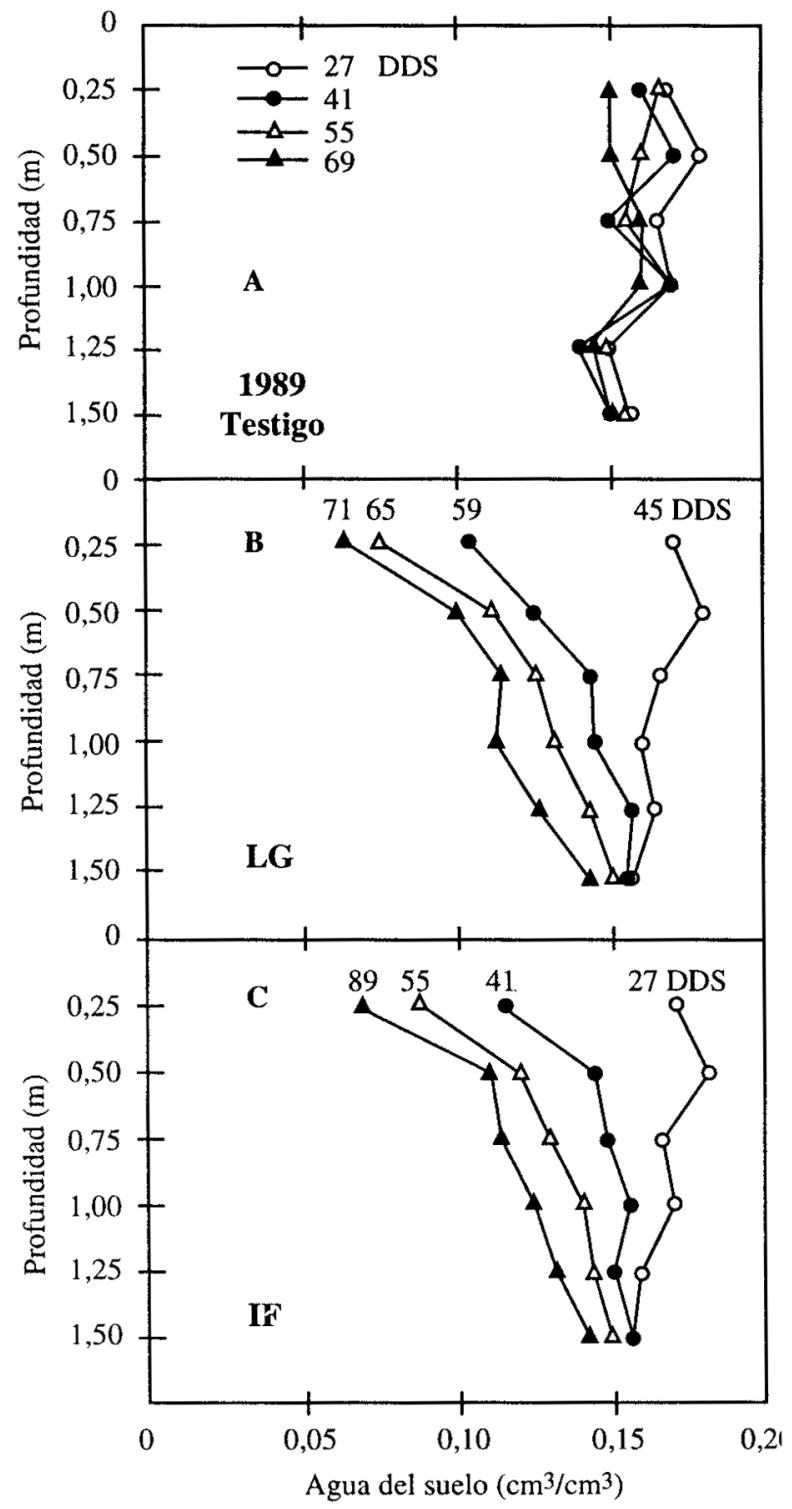

Figura 2. Cambios en el contenido volumétrico de agua en los tratamientos de sequía iniciados en la etapa de llenado de grano (LS) y f1oración (IF) en contraste con el testigo. KBS, Michigan.

testigo y en el tratamiento IF. La conductancia estomática observada a los 36 DDS fue menor en IF que en el testigo (Figura 4). Esta diferencia fue significativa también a los 46 DDS pero no a los 55 DDS. Durante 1989, la conductancia estomática disminuyó drásticamente en las mediciones hechas a los 42 y 52 DDS alcanzando valores por debajo de $0,06 \mathrm{~cm} / \mathrm{seg}$. Esto obedece aparentemente, a que en esas dos fechas se tuvieron días calurosos (para este tipo de clima) con temperaturas por arriba de $\operatorname{los} 30^{\circ} \mathrm{C}$. Turner y Kramer (1980), reportaron cierres estomáticos para diferentes cultivos provocados por la alta demanda atmosférica por agua observada du-

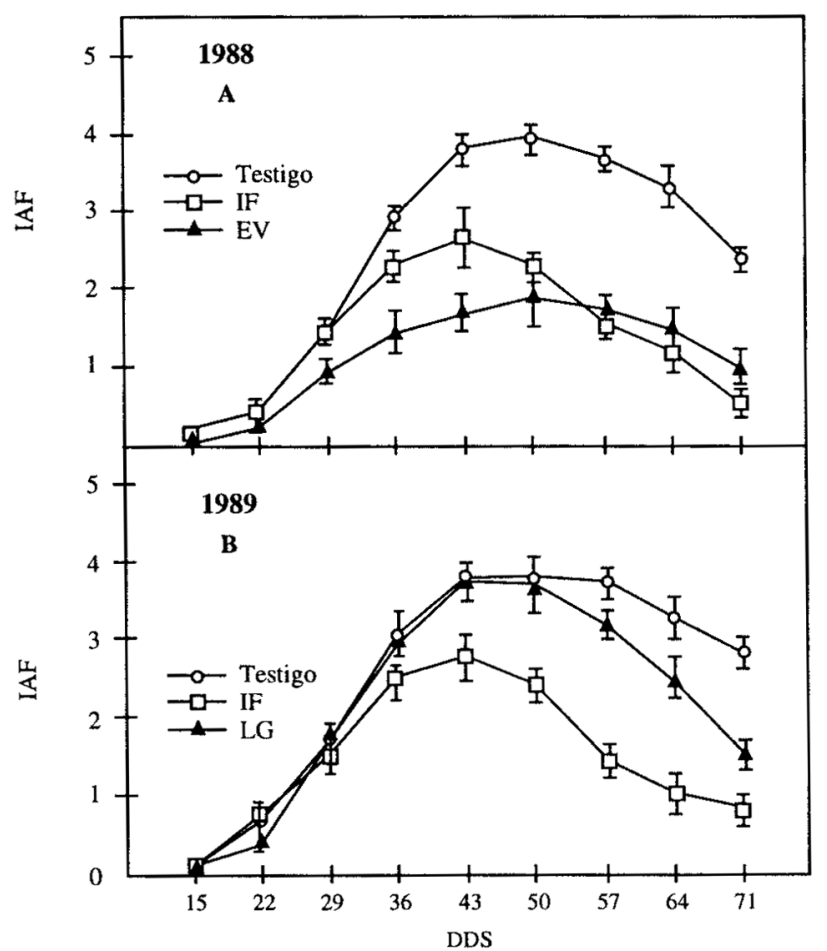

Figura 3. Dinámica de crecimiento en el índice de área foliar de frijol bajo diferentes condiciones de humedad en el suelo. KBS, Michigan. Las barras representan el error estándar.

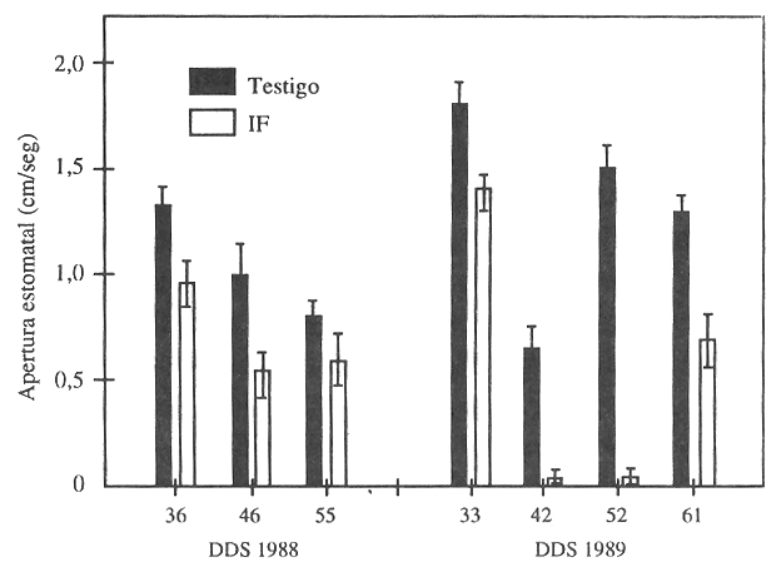

Figura 4. Conductancia estomática en el frijol común durante la floración y el llenado de semillas bajo condiciones de riego y sequía. KBS, Michigan las barras representan el error estándar.

rante el medio día. En el presente experimento el cierre de los estomas a los 42 y 52 DDS causó una disminución en las tasas de asimilación de $\mathrm{CO}_{2}$ donde la fotosíntesis decreció en un 60-80 \% en comparación con el 


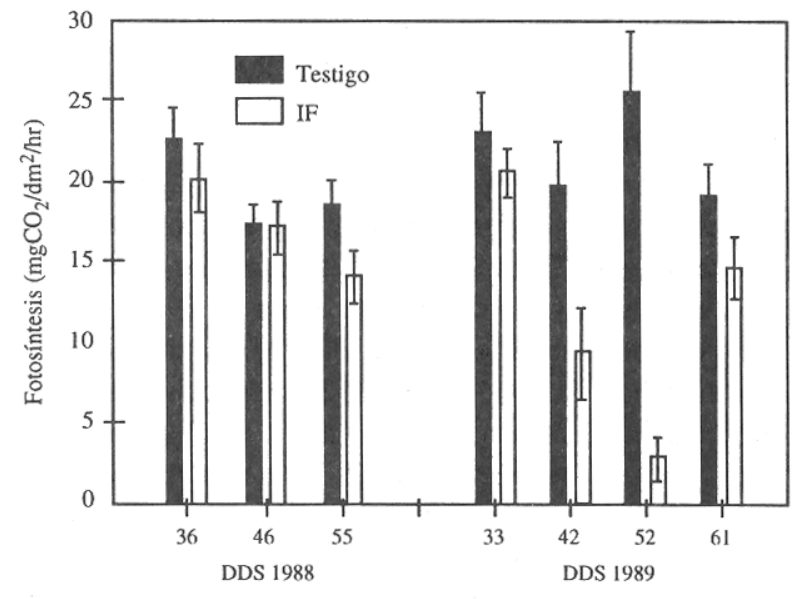

Figura 5. Tasas de fotosíntesis en frijol durante floración y llenado de la semilla bajo condiciones de riego y sequía. KBS, Michigan. Las barras representan el error estándar.

testigo. Sin embargo, las diferencias se redujeron hacia el final de la estación de crecimiento como se observa en las mediciones hechas 61 DDS (Figura 5).

El efecto de la conductancia estomática sobre la fotosíntesis (Pn) se describe mejor en la Figura 6. Para el tratamiento bajo riego hay un incremento de $\mathrm{Pn}$ al pasar de aperturas estomáticos de 0,7 a $1,8 \mathrm{~cm} / \mathrm{seg}$ alcanzando un valor máximo de $25 \mathrm{mg} \mathrm{CO} / \mathrm{dm}^{2} / \mathrm{hr}$. Bajo el tratamiento de estrés, la máxima tasa de fotosíntesis observada fue de cerca de $20,0 \mathrm{mg} / \mathrm{CO}_{2} / \mathrm{dm}^{2} / \mathrm{hr}$. Las tasas de fotosíntesis no fueron diferentes significativamente entre tratamientos en el ámbito de conductancia estomática entre 0,6 y 1,4 cm/seg. Schultz y Hall (1982), encontraron que al imponer tratamientos de sequía a plantas C3, generalmente éstas disminuían su conductancia estomática antes de disminuir las tasas de asimilación de $\mathrm{CO}_{2}$. Lynch et al. (1992) también encontraron, en diferentes variedades de frijol, que la apertura estomática no estuvo muy relacionada con las tasas de fotosíntesis sino con el contenido de nitrógeno y clorofila en las hojas. También Castanguay y Maskhart (1991) mostraron que la capacidad fotosintética del mesófilo no es fácilmente afectada aún en variedades no resistentes a sequía.

En comparación con el testigo y la intercepción de luz en el tratamiento IF disminuyó en un $26 \%$ a los 46 DDS. Después de esta fecha, se observó un decremento significativo en la cantidad de luz interceptada en IF debido principalmente a la cantidad de área foliar perdida por senescencia (Figura. 7). Entre 46 y 60 DDS la intercepción de luz disminuyó de $63 \%$ a $24 \%$ y para la parte final de la estación de crecimiento la intercepción

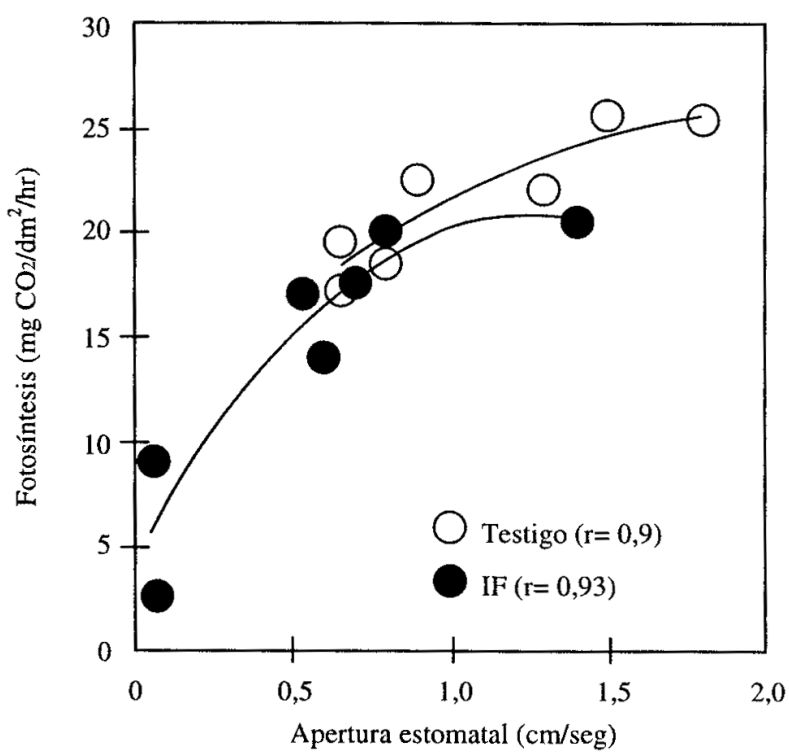

Figura 6. Relación entre la conductancia estomática y la fotosíntesis en frijol bajo condiciones de riego y sequía. KBS, Michigan.

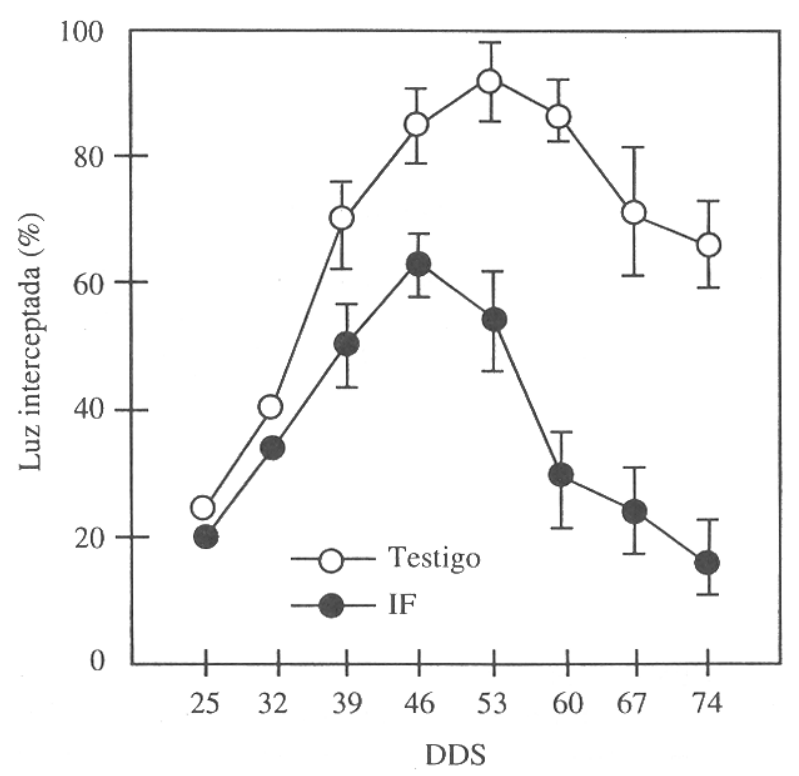

Figura 7. Cambios estacionales en la intercepción de luz medida al medio día en los tratamientos de riego y sequía. KBS, Michigan. Las barras representan el error estándar.

de luz fue de $16 \%$ comparada con un $66 \%$ de luz interceptada en el testigo. Foster et al. (1995) encontró que en frijol bajo sequía, las altas tasas de senescencia foliar se deben principalmente ha una rápida translocación de nitrógeno a los órganos reproductivos. 
Por otro lado, la producción de biomasa estuvo altamente relacionada con el porcentaje de energía solar interceptada (Figura 8). La misma relación a sido encontrada para diferentes cultivos como cebada, papa, remolacha y trigo (Monteith, 1977, Gallagher y Biscoe, 1978). Sin embargo, al comparar el tratamiento de sequía IF con el testigo se observa un diferencia de $6,5 \%$ la acumulación de materia seca al 50\% de luz interceptada. Esta diferencia puede deberse a un incremento en la translocación de carbohidratos a la raíz durante el periodo de deficiencia hídrica. Hoogenboom, et al. (1987), encontraron que durante períodos de sequía el crecimiento de la raíz se incrementa a expensas del crecimiento de la parte aérea.

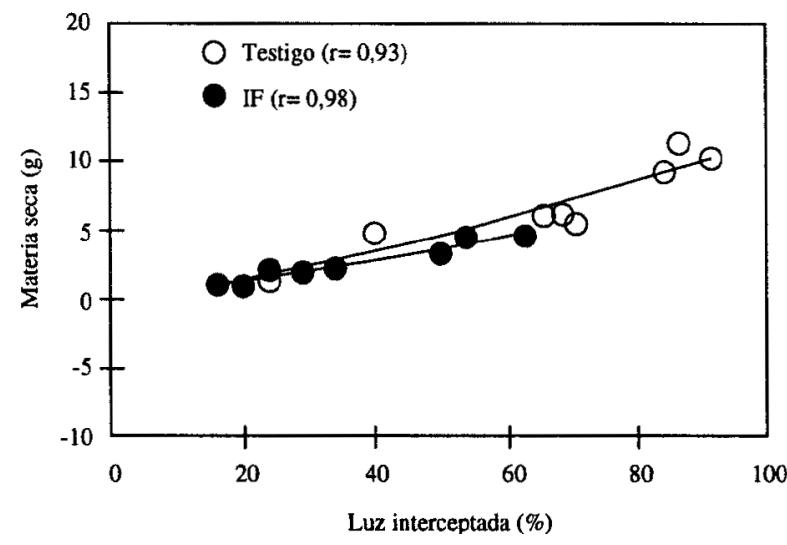

Figura 8. Relación entre porcentaje de luz interceptada y producción de materia seca en frijol en riego y sequía. KBS, Michigan.
Nuñez-Barrios (1991), también encontró que en frijol bajo sequía, se duplicaba el número de raíces secundarias y terciarias al mismo tiempo que se reducen las tasas de crecimiento foliar.

La producción total de materia seca y peso de semillas disminuyó significativamente en los tratamientos de sequía (Cuadro 1). En el tratamiento IF se redujo el peso seco total de la parte aérea en 69,9 y 72,0\% y el peso de la semilla en 77,8 y 79,1\% en comparación con el testigo durante 1988 y 1989 respectivamente. La producción de materia seca en EV y LS disminuyó en 60,8 y 43,0\% respectivamente. También el índice de cosecha diminuyó considerablemente para los tratamientos de sequía, siendo el tratamiento IF el más afectado ya que la sequía comenzó justo al inicio de la formación de flores.

Los tratamientos de sequía en frijol disminuyeron la conductancia estomática y en menor intensidad las tasas de fotosíntesis, sin embargo el índice de área foliar fue reducido significativamente por el déficit hídrico impuesto, debido principalmente a una disminución en el crecimiento foliar en el tratamiento EV y a un aumento en las tasas de senescencia en los tratamientos IF y LS.

La producción de materia seca en la parte aérea de la planta estuvo altamente relacionada con la intercepción de la radiación fotosintéticamente activa, aunque al mismo valor de intercepción de luz, el tratamiento de sequía alcanzó a producir una cantidad de materia seca ligeramente menor en la parte aérea en comparación con el testigo, sugiriendo un incremento en la translocación de carbohidratos a la raíz a medida que disminuye la disponibilidad de agua en el suelo.

Cuadro 1. Peso total de materia seca de la parte aérea, producción de semilla e índice de cosecha del frijol en diferentes tratamientos de humedad del suelo. KBS, Michigan.

\begin{tabular}{rlccc}
\hline Año & Tratamiento & $\begin{array}{c}\text { Peso seco } \\
\text { Total }(\mathbf{k g} / \mathbf{h a})\end{array}$ & $\begin{array}{c}\text { Peso de semillas } \\
(\mathbf{k g} / \mathbf{h a})\end{array}$ & $\begin{array}{c}\text { Indice de } \\
\text { cosecha }\end{array}$ \\
\hline \multirow{2}{*}{1988} & Testigo & 4.868 & 1.850 & 0,38 \\
& Sequía IF & 1.467 & 411 & 0,28 \\
& Sequía EV & 1.906 & 572 & 0,30 \\
& DMS $(\alpha=0,05)$ & 328 & 256 & \\
1989 & & & & \\
& Testigo & 4.884 & 1.905 & 0,39 \\
& Sequía LS & 2.776 & 913 & 0,33 \\
& Sequía IF & 1.372 & 398 & 0,29 \\
& DMS $(\alpha=0,05)$ & 575 & 492 & \\
\hline
\end{tabular}




\section{LITERATURA CITADA}

ADAMS, J.; ARKIN, G. 1977. A light interception method for measuring row crop ground cover. Soil Sc. Soco Am. J. 41: 789-792.

CASTONGUAY, Y; MARKHART, A.H. 1991. Saturated rates of photosynthesis in water-stressed leaves of common beans and tepary beans. Crop Sc. 31: 1605-1611.

CONGRESO NACIONAL DE BIOTECNOLOGIA. (1996, MEXICO). Degradación de clorofila y pérdida de almidón en frijol bajo sequía. Publicado por Nuñez-Barrios, A y Rumayor, A., Chihuahua, Mex., ANABAF. 1 v.

DAI, Z.; EDWARDS, G.E.; KU, M.S. 1992. Control of photosynthesis and stomatal conductance in castor bean by leaf to air vapor pressure deficit. Plant Physiol. 99: $1426-1434$.

FARQHUAR, G.D.; SHARKEY, T.D.1982. Stomatal conductance and photosynthesis. Ann. Review Plant Physiol. 33:317-345.

FOSTER, E.; PAJARITO, A.; ACOSTA, 1. 1995. Moisture stress impact on $\mathrm{N}$ partitioning, $\mathrm{N}$ remobilization and $\mathrm{N}$-use efficiency in beans. J. Agri. Sci. 124:27-37.

GALLAGHER, J.N.; BISCOE, P.v. 1978. Radiation absorption, growth and yield of cereals. J. Agric. Sci. Camb. 91:47-60.

HALL, A.E.; SHULTZ, E.D. 1980. Stomatal response to environment and a possible interrelation between stomatal effect on transpiration and $\mathrm{CO}_{2}$ assimilation. Plant Cell Environ. 3: 467-474.

HOOGENBOOM, G.; HUCK, M.G.; PETERSON, e.M. 1987. Root growth rate of soybean as affected by drought stress. Agron. J. 79:607-614.
KRAMER, P.J. 1983. Water relations of plants. Academic Press Inc, N.Y. 389 p.

LAING, D.R.; KRETCHMER, P.; ZULUAGA, S.; JONES, P.G. 1983. Field Beans. Ed.por W.H. Smith, Los Baños, Philippines, IRRI. 248 p.

LYNCH, J.; GONZALEZ, A.; TOHME, J.; GARCIA, 1. 1992. Variation in characters related to leaf photosynthesis in wild beans populations. Crop Sci. 32:633-640.

MONTHEITH, J.L. 1977. Climate and efficiency of crop production. Phil. Trans. Roy. Soco London. 281:277-294.

MORGAN, J.M. 1984. Osmoregulation and water stress in higher plants. Ann. Rev. Plant Physiol. 35:299-319.

NESMITH, D.S.; MILLER, A.; RITCHIE, J. 1990. An irrigation system for plots under a rain shelter. Agric. Water Mgmt. 17:409-414

NUÑEZ-BARRIOS, A. 1991. Effect of soil water deficits on the growth and development of dry bean at different stages of growth. PhD. Thesis, Michigan State University $128 \mathrm{p}$.

NUÑEZ-BARRIOS, A.; FOSTER, E. 1996. Efecto del déficit hídrico sobre el crecimiento de hojas, tallos y vainas de frijol. Agricultura Tec. en México 22:99-109

SHULTZ, E.D.; HALL, A.E. 1982. Stomatal responses, water loss and $\mathrm{CO}_{2}$ assimilation rates of plants in contrasting environments. Encycl. Plant Physiol. 12B: 181-230.

TURNER, N.C.; KRAMER, P.J. 1980. Adaptation of plants to water and high temperature stress. Wiley Interscience, N.Y. 387p. 
